\title{
A Comparison Between Foley and Nelatone Urinary Catheters in Causing Urinary Tract Infection in Animal Models
}

\author{
Saeed Nouri ${ }^{1}$; Mohammad Reza Sharif ${ }^{2, *}$; Mehrdad Hosseinpour ${ }^{2}$; Shima Farokhi ${ }^{3}$; \\ Mohammad Hossein Sharif ${ }^{4}$ \\ ${ }^{1}$ Chemical Injuries Research Center, Baqiyatallah University of Medical Sciences, Tehran, IR Iran \\ ${ }_{2}^{2}$ Trauma Research Center, Kashan University of Medical Sciences, Kashan, IR Iran \\ ${ }^{3}$ Department of Community Health Nursing, Hamadan University of Medical Sciences, Hamadan, IR Iran \\ ${ }^{4}$ Department of Biomedical Engineering, Science and Research Branch, Islamic Azad University, Tehran, Iran \\ ${ }^{*}$ Corresponding author: Mohammad Reza Sharif, Trauma Research Center, Kashan University of Medical Sciences, Kashan, IR Iran. Tel: +98-9123788713, Fax: +98-3155548900, E-mail: \\ mrsharifmd@yahoo.com
}

Received: October 6, 2014; Revised: January 1, 2015; Accepted: January 13, 2015

Background: Urinary catheterization is one of the main measures used to treat and care for hospitalized patients. Several complications have been attributed to the presence of latex with routine Foley catheters. Therefore, some studies have recommended that Nelatone catheters be substituted for the ordinary Foley catheters to prevent these complications.

Objectives: This study aimed to compare the rates of urinary tract infection(UTI) in rabbits catheterized either with Foley or with Nelatone catheters.

Materials and Methods: A randomized controlled trial was conducted on 60 rabbits that were randomly assigned to three groups of twenty. The first group was catheterized using Nelatone catheter; the second group was catheterized using Foley catheter and the third group was studied without performing any catheterization. After seven days, urine samples were collected using suprapubic aspiration and were sent to the laboratory for culture. Descriptive statistics were calculated. Moreover, chi-square and Fisher's exact tests were used for data analysis.

Results: At the end of the study, four 4 cases in the Nelatone group and 12 cases in the Foley group presented with UTI ( $P=0.01)$. No positive urine cultures were found in the control group.

Conclusions: The Nelatone catheters, compared with the Foley ones, had a lower risk of UTI in the long term use. Verifying this claim by further studies can have an important role in reducing UTIs in patients using urinary catheters.

Keywords: Urinary Tract Infection; Urinary Catheterization; Catheters; Rabbit

\section{Background}

In several studies carried out on the incidence of nosocomial infections, urinary tract infections (UTI) were the most frequent and consisted more than $40 \%$ of all hospital infections (1-7). In spite of the broad spectrum antibiotics use, UTIs are still a cause of considerable morbidity and mortality in the modern medicine. UTIs are usually associated with urinary catheters (UC) or urologic procedures (8-10). Urinary catheterization is one of the main measures used for the treatment and caring for hospitalized patients. It is associated with the emergence of bacteria in the urine, unpleasant symptoms, and complications that can reduce the quality of health care services (11). Despite considerable advancements in manufacturing medical devices such as UCs, infections caused by these devices are common, costly, and can cause significant patient morbidity $(2,12)$.

The pathogenesis of infections caused by medical devices is not well understood and a variety of factors are considered such as susceptibility of the host and the mi- croorganisms' ability to attach to the medical devices (8). Biofilm formation along the catheter surface is the most important cause of bacteriuria. Biofilm formation begins immediately after catheter insertion, when organisms adhere to a conditioning film of host proteins which forms along the catheter surface. Both the interior and exterior catheter surfaces are involved. Bacteria usually originate from the periurethral area or ascend the drainage tubing following colonization of the drainage bag (13). There are different types of UCs in terms of their materials, sizes and shapes and each of them is made for a specific purpose. The aim of this diversity is to reduce the risk of UTI $(4,14)$. UCs are also categorized considering their location and duration of use (15). Due to the fact that in some patients, such as those admitted to the intensive care units, the UCs should long remain in the bladder, a variety of specialized antiseptic/antibiotic impregnated catheters have been designed to reduce the risk of UTI; but they are expensive and not available in some medical

Copyright (C) 2015, Kashan University of Medical Sciences. This is an open-access article distributed under the terms of the Creative Commons Attribution-NonCommercial 4.0 International License (http://creativecommons.org/licenses/by-nc/4.0/) which permits copy and redistribute the material just in noncommercial usages, provided the original work is properly cited. 
centers (16) and some studies showed these catheters did not reduce symptomatic UTI (17).

Therefore many specialists use ordinary Foley catheters in these circumstances (14). However, due to the existence of latex with these catheters, changes in bladder tissue, infections, and in some cases hemorrhagic cystitis has been reported. Thus, in some studies it has recommended that Nelatone catheters should be substituted for ordinary Foley catheters to prevent these complications (18).

\section{Objectives}

Due to the fact that there are no studies to compare the complications of these two types of UCs according to the laboratory tests and urine cultures, this study aimed to compare the rates of UTI in rabbits catheterized either with Foley or Nelatone catheters.

\section{Materials and Methods}

This experimental study was conducted at Kashan University of Medical Sciences from July to September 2013. In this study, 60 five-months-old New Zealand male rabbits weighing 2 to 2.5 kilograms were randomly assigned to three groups of twenty. One week before study, animals were fed similarly and were kept at $21 \pm 1^{\circ} \mathrm{C}$ with 12 hour light/dark cycle (lights on from 8:00 AM to 8:00 PM). Twenty rabbits were catheterized using Nelatone catheters (No: 8, made by VRP Co, Malaysia); the second group were catheterized using Foley catheters (No: 8, made by VRP Co, Malaysia). Rabbits in the third group were selected as control group and were not catheterized. For catheterization, the animal was placed in a horizontal dorsal decubitus position on the surgical table and its paws were fixed to the extremities of the table with thin ropes. The catheters were installed by a surgery resident using correct technique of aseptic catheter insertion and measures for instant, adhesive fabric for fixation and use of lubricant gel for inserting the catheter into urethra were carefully performed under sterile condition. The catheters were kept inside the bladder for seven days. Then urine samples (i.e. $1 \mathrm{~mL}$ ) were collected from all the three groups through suprapubic method under aseptic conditions. The specimens were immediately sent to the laboratory and cultured on blood agar medium. Based on the result of urine cultures, a UTI was diagnosed when one or more organisms were present at quantitative counts of greater than or equal to 100 colony-forming units (CFU) per milliliter. Then the causative organisms were determined. All data were documented in a checklist consisting items on the name of group, the code of rabbit, the number of CFU in the urine culture and the type of the bacteria grew in the culture medium.

\subsection{Ethical Considerations}

Animal handling and all experiments were performed in accordance with the international guidelines set out in the Guide for the Care and Use of Laboratory Animals (Institute of Laboratory Animal Resources, 1996) (19) and the Helsinki declaration has been respected. The study protocol was approved by the local research council and the research ethics committee at Kashan University of Medical Sciences, Kashan, Iran.

\subsection{Data Analysis}

All information was extracted from data registration forms and the data were entered into SPSS 13.0 software. Chi-square and Fisher's exact tests were used for data analysis. Statistical significance was considered at $P$ value $<0.05$.

\section{Results}

No positive urine cultures were found in the control group. The number of UTI werefour cases in the Nelatone group and 12 cases in the Foley group $(P=0.01)$ (Table 1 ).

The frequency of entrococcal infection was less in the Nelatone group (3 cases) compared with the Foley group (nine cases) and this difference was statistically significant $(\mathrm{P}=0.038)$. Moreover, three cases of staphylococcal UTI were observed in the Foley group compared to only one case in the Nelatone group $(\mathrm{P}=0.605)$ (Table 2$)$.

Table 1. The Numbers of Urinary Tract Infection in the Nelatone Group and the Foley Group ${ }^{a}$

\begin{tabular}{lccc}
\hline & \multicolumn{2}{c}{ Groups } & P Value $^{\mathrm{b}}$ \\
\cline { 2 - 3 } & Nelatone & Foley & \\
\hline Urinary tract infection & & & \\
No & $16(80)$ & $8(40)$ & 0.01 \\
Yes & $4(20)$ & $12(60)$ & 0.01 \\
\hline
\end{tabular}

${ }_{\mathrm{a}}$ All Values are Presented as No. (\%).

b Chi Square Test.

Table 2. The Frequency of Organism in the Nelatone Group Compared with the Foley ${ }^{\mathrm{a}}$

\begin{tabular}{|c|c|c|c|}
\hline \multirow{2}{*}{$\begin{array}{l}\text { Bacteria Isolated from } \\
\text { the Urine Culture }\end{array}$} & \multicolumn{2}{|c|}{ Groups } & \multirow[t]{2}{*}{ P Value } \\
\hline & Foley & Nelatone & \\
\hline Entrococcal Infection & & & $0.038^{b}$ \\
\hline No & $11(55)$ & & \\
\hline Yes & $9(45)$ & & \\
\hline $\begin{array}{l}\text { Staphylococcal } \\
\text { infection }\end{array}$ & & & $0.605^{c}$ \\
\hline No & $17(85)$ & $19(95)$ & \\
\hline Yes & $3(15)$ & 1(5) & \\
\hline
\end{tabular}




\section{Discussion}

In this study, UTI was observed in the both intervention groups but no infection was observed in the control group. There were significant differences between the two types of catheters in causing UTI and the Nelatone catheter was associated with a lower risk of UTI. Few studies have published in this field. These studies only assessed the risk of developing UTI after inserting a UC and have reported conflicting results. Uckay et al. have studied 8169 patients with UCs and reported that the long-term exposure to a UC is a definite risk factor for development of UTI. They suggest that further investigation is warranted to determine the other factors affecting UC associated UTIs (20). Consistent with results of the present study, Uckay et al. confirmed that a higher rate of UTI should be expected in patients who undergo urinary catheterization. However, they did not investigate the difference in the rates of UTI in patients with different types of UCs. On the other hand, Smarick et al. studied the incidence of catheter-associated UTI in dogs and reported that placement of an indwelling UC is associated with a low risk of UTI in dogs. They also reported that the results of bacterial culture of UC tips cannot predict the development of catheter-associated UTIs (21). Hosseinpour et al. (16) and Nicolle (13) have also observed no significant difference between silicone and latex catheters regarding the risk of UTI. This finding is similar to Schumm et al. (4) who reported that there is not enough evidence on superiority of different types of catheters on each other considering the risk of UTI in hospitalized patients with short-term catheterization. These studies along with our results show the necessity to determine the factors affecting the rate of TUIs in catheterized patients.

The most effective way to prevent infection is to limit catheter use and discontinue the catheter when no longer needed. Catheter removal or exchange might also be useful in management of UTI associated with UCs (7). Nurses can help to prevent catheter-associated UTIs through using aseptic technique on insertion, follow best practice in ongoing care and prompt removing of catheters (22).

Some researchers indicate that antibiotic prophylaxis significantly reduced the rate of catheter-associated UTIs $(23,24)$ but guidelines do not recommend routine use of antibiotics $(23,25)$ or antimicrobial coated catheters (13, 25) to prevent catheter-associated UTIs.

In the present study, the microorganisms isolated from the rabbits with UTI, were enterococcus and staphylococcus. However, in a study on patients with symptomatic UTI, Escherichia coli (E. coli), Klebsiellapneumoniae, Pseudomonas aeruginosa, Acinetobacterspp, Staphylococcus, Enterococcus and Gram-negative bacteria were the most common isolated organisms (26). In two other human studies, E. coli was the predominant organism isolated from catheterized patients $(27,28)$. Nonetheless, in an animal study, E. coli and Proteus spp. were more frequent in non- catheterized dogs, whereas Enterobacter spp. and Staphylococcus spp. were more frequent in catheterized dogs (29). Evidence show that the patients' skin and high-touch environmental surfaces are important sources for contamination of UCs in place, in hospitalized patients (30).

In this study, we conducted an investigation to identify the most appropriate catheter (Foley vs. Nelatone) for short-term urinary catheterization in animal model. We did not evaluate bladder histological changes in our animals and this would be one of the weaknesses of the present study. In conclusion, there was a significant difference between Nelatone and Foley catheters in producing UTIs. Using Nelatone catheters bear a far lower risk of infection. Considering the high prevalence of UTI after long-term use of a UCs in hospitalized patients and the subsequent prolonged hospitalization and health care financial burdens, it can be suggested that Nelatone catheters may be used instead of Foley ones. Further investigation is warranted to determine the effectiveness of the type of the UC and the appropriate type of catheter for each patient.

\section{Acknowledgements}

The authors would like to appreciate the kind assistance and financial support provided by the Vice-Chancellor for Research at Kashan University of Medical Sciences.

\section{Funding/Support}

This study was supported financially by the Vice-Chancellor for Research of Kashan University of Medical Sciences (Grant number: 9067).

\section{References}

1. Foxman B. Urinary tract infection syndromes: occurrence, recurrence, bacteriology, risk factors, and disease burden. Infect Dis Clin North Am. 2014;28(1):1-13.

2. Chenoweth CE, Gould CV, Saint S. Diagnosis, management, and prevention of catheter-associated urinary tract infections. Infect Dis Clin North Am. 2014;28(1):105-19.

3. Esposito S, Noviello S, Leone S. [Catheter-associated urinary tract infections: epidemiology and prevention]. Infez Med. 2008;16(3):130-43.

4. Schumm K, Lam TB. Types of urethral catheters for management of short-term voiding problems in hospitalized adults: a short version Cochrane review. Neurourol Urodyn. 2008;27(8):738-46.

5. Lam TB, Omar MI, Fisher E, Gillies K, MacLennan S. Types of in dwelling urethral catheters for short-term catheterisation in hospitalised adults. Cochrane Database Syst Rev. 2014;9:CD004013.

6. Gardner A, Mitchell B, Beckingham W, Fasugba O. A point prevalence cross-sectional study of healthcare-associated urinary tract infections in six Australian hospitals. BMJ Open. 2014;4(7):e005099.

7. Al Mohajer M, Darouiche RO. Prevention and treatment of urinary catheter-associated infections. Curr Infect Dis Rep. 2013;15(2):116-23.

8. Elder JS. Urinary Tract Infections. 2011:1829-340.

9. Kronman MP, Sherilyn Smith S. Infectious Diseases. 7th ed. Marcdante KJ, Kliegman RM editors. Philadelphia: Elsevier Saunders; 2015.

10. Nguyen HI. Bacterial infection of the genitourinary tract. 18 edNew York: McGraw Hill; 2013. 
11. Kheyrkhah H, Ramezani K, Mirkarimi T, Ghavam M. Steriled and Nonsteriled Urinary Catheters and the Urinary Tract Infection in Hospitalized Patients. Arch Clin Infect Dis. 2013;8(4).

12. Adib-Hajbaghery M, Aghajani M. Quality of care for patients with indwelling urinary catheter in selected hospitals in Kashan, Iran 2007. Int J Urol Nurs. 2009;3(2):43-9.

13. Nicolle LE. Catheter associated urinary tract infections. Antimicrob Resist Infect Control. 2014;3:23.

14. Leuck AM, Wright D, Ellingson L, Kraemer L, Kuskowski MA, Johnson JR. Complications of Foley catheters-is infection the greatest risk? J Urol. 2012;187(5):1662-6.

15. Zinner M, Ashley SW. Maingot's abdominal operations. 12th edNew York: McGraw Hill; 2013.

16. Hosseinpour M, Noori S, Amir-Beigi M, Pourfakharan MH, Ehteram H, Hamsayeh M. Safety of latex urinary catheters for the short time drainage. Urol Ann. 2014;6(3):198-201.

17. Makuta G, Chrysafis M, Lam T. Measuring the efficacy of antimicrobial catheters. Nurs Times. 2013;109(44):16.

18. Stoller ML. Retrograde instrumentation of the urinary tract. 18 ed. McAninch J, Lue TF editors. New York: McGraw Hill; 2013.

19. Committee for the update of the guide for the care and use of laboratory animals.. Guide for the Care and Use of Laboratory Animals. 8th edWashington(DC): The national academies press;2011.

20. Uckay I, Sax H, Gayet-Ageron A, Ruef C, Muhlemann K, Troillet N, et al. High proportion of healthcare-associated urinary tract infection in the absence of prior exposure to urinary catheter: a cross-sectional study. Antimicrob Resist Infect Control. 2013;2(1):5.

21. Smarick SD, Haskins SC, Aldrich J, Foley JE, Kass PH, Fudge M, et al. Incidence of catheter-associated urinary tract infection among dogs in a small animal intensive care unit. J Am Vet Med Assoc. 2004;224(12):1936-40.
22. Dailly S. Prevention of indwelling catheter-associated urinary tract infections. Nurs Older People. 2011;23(2):14-9.

23. Pereira S, Nguyen L, Stevermer JJ. PURLs: A simple way to reduce catheter-associated UTIs.J Fam Pract. 2014;63(5):E10-2.

24. Lo E, Nicolle LE, Coffin SE, Gould C, Maragakis LL, Meddings J, et al. Strategies to prevent catheter-associated urinary tract infections in acute care hospitals: 2014 update. Infect Control Hosp Epidemiol. 2014;35(5):464-79.

25. Gould CV, Umscheid CA, Agarwal RK, Kuntz G, Pegues DA, Healthcare Infection Control Practices Advisory C. Guideline for prevention of catheter-associated urinary tract infections 2009. Infect Control Hosp Epidemiol. 2010;31(4):319-26.

26. Niveditha S, Pramodhini S, Umadevi S, Kumar S, Stephen S. The Isolation and the Biofilm Formation of Uropathogens in the Patients with Catheter Associated Urinary Tract Infections (UTIs). $J$ Clin Diagn Res. 2012;6(9):1478-82.

27. Melzer M, Welch C. Outcomes in UK patients with hospital-acquired bacteraemia and the risk of catheter-associated urinary tract infections. Postgrad Med J. 2013;89(1052):329-34.

28. Ortega M, Marco F, Soriano A, Almela M, Martinez JA, Pitart C, et al. Epidemiology and prognostic determinants of bacteraemic catheter-acquired urinary tract infection in a single institution from 1991 to 2010. J Infect. 2013;67(4):282-7.

29. Bubenik LJ, Hosgood GL, Waldron DR, Snow LA. Frequency of urinary tract infection in catheterized dogs and comparison of bacterial culture and susceptibility testing results for catheterized and noncatheterized dogs with urinary tract infections. J Am Vet Med Assoc. 2007;231(6):893-9.

30. Linder KA, Hecker MT, Kundrapu S, Cadnum JL, Musuuza JS, Sethi AK, et al. Evaluation of patients' skin, environmental surfaces, and urinary catheters as sources for transmission of urinary pathogens. Am J Infect Control. 2014;42(7):810-2. 